\title{
ESTUDIO DE LA HIDRÓLISIS ÁCIDA DEL RAQUIS DE BANANO
}

\section{ACID HYDROLYSIS OF BANANA RACHIS STUDY}

\author{
Anderson Guarnizo Franco ${ }^{1}$, Pedro Nel Martínez Yepes ${ }^{2}$
}

\author{
1. Profesor programa Química de la Universidad del Quindío, aguarnizo@uniquindio.edu.co \\ Profesor Titular programa de Química de la Universidad del Quindío. \\ Fecha de recibido: Octubre 28 de 2010 \\ Fecha de aceptado: Agosto 15 de 2011 \\ Correspondencia: Programa de Química, Universidad del Quindío. Av. Bolívar calle 12 norte Armenia Quindío Colombia
}

\section{RESUMEN}

La hidrólisis ácida es un método de pretratamiento que ha sido empleado para aumentar la accesibilidad de la biomasa frente a un ataque enzimático en los procesos que conllevan a la obtención etanol. Por tal motivo, el efecto de hidrólisis ácida sobre la biomasa lignocelulósica del raquis de banano Cavendish Valery fue investigado. Usando el parámetro de severidad combinada (CS), que involucra la variable temperatura, tiempo y pH, se observó como varió el contenido de materia seca, celulosa, holocelulosa, hemicelulosa, azúcares reductores y 5hidroximetilfurfural (HMF). Los resultados registran el momento en que se logra una mayor concentración de azúcares reductores y una producción despreciable de HMF cuando la CS es de 2,67 (170 ㄷ, 40 min y 1\% de ácido sulfúrico). Además, en estas condiciones, el material muestra una avanzada degradación de hemicelulosa, mientras que no se evidenció el mismo efecto sobre la celulosa.

Palabras clave: raquis, banano, etanol, pretratamiento, hidrólisis ácida.

\begin{abstract}
Acid hydrolysis is a pretreatment method used in order to enhance biomass accessibility to enzymatic hydrolysis towards ethanol production. For that reason, some chemical changes in biomass from Cavendish Valery banana rachis by acid hydrolysis were researched. Using the combined severity (CS) that takes into account temperature, time and $\mathrm{pH}$; how the change in the content of cellulose, holocellulose, hemicelulose, reducing sugars and 5-

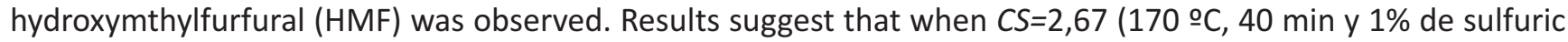
acid), the sample showed a maximum sugar content versus low HMF formation. Additionally, hemicelluloses degradation was established meanwhile cellulose degradation was not evident.
\end{abstract}

Keywords: Rachis, banana, ethanol, pretreatment, acid hydrolysis.

\section{INTRODUCCIÓN}

Debido al eventual agotamiento de los recursos energéticos no renovables, principalmente los derivados del petróleo, el mundo ha volcado la mirada hacia nuevas fuentes de energía renovables(1), especialmente los recursos lignocelulósicos residuales derivados de la actividad agrícola pues representan una fuente abundante, continua y económica, útil para una industria basada en la biorefinación (2).

En relación a estas nuevas fuentes, hasta el 2007, Colombia tenía cultivada aproximadamente 420 mil ha de diferentes Musacea. Esta actividad genera una considerable cantidad de residuos, pues solo el $12 \%$ del peso de la planta es aprovechado como fruto comestible, el resto comprende el componente fibroso y no fibroso que incluye el raquis (eje del racimo de banano), el pseudotallo y las hojas (3). Usualmente, este tipo deresiduos, están constituidos principalmente por holocelulosa, que corresponde casi siempre a más del $50 \%$ del peso seco de la biomasa. La holocelulosa es un agregado heterogéneo de carbohidratos, compuesto por celulosa y hemicelulosa integrados a la pared celular en conjunto con la lignina $(4,5)$. La biomasa resulta ser un recurso natural importante debido a que la holocelulosa puede ser degrada a sus constituyentes monoméricos (azúcares)y 
posteriormente convertida en etanol. Aunque la transformación de la celulosa y hemicelulosa en monosacáridos resulta sencillamente de la hidrólisis de los enlaces glucosídicos, la disposición de los constituyentes de la pared celular (celulosa, hemicelulosa y lignina), convierte a la biomasa en un material excepcionalmente resistente a los ataques químicos en condiciones moderadas de reacción que pueden dar lugar a la liberación de los azúcares. Por esta razón, actualmente aún se debate la estrategia metodológica que logre liberar los monosacáridos útiles en la fermentación (2). Por lo regular, la estrategia involucra un pretratamiento que logre retirar a la lignina y/o la hemicelulosa dejando a la celulosa accesible a ataques enzimáticos y luego, una vez se ha asegurado la formación de monosacáridos, los hidrolizados son sometidos a fermentación alcohólica. Siendo el pretratamiento considerado como la etapa crítica en la conversión de la biomasa en etanol (6) son varias las tecnologías que han sido ensayadas (7), entre estas, está la hidrólisis ácida a baja concentración. La hidrólisis con ácido a baja concentración involucra el uso de un ácido (usualmente ácido sulfúrico) bajo ciertas condiciones de temperatura y tiempo, para remover la hemicelulosa. Paralelamente, mientras se hace un pretratamiento de hidrólisis ácida, se puede formar subproductos de degradación de carbohidratos (principalmente 5-HMF o HMF) en parte responsable de la inhibición en la etapa de la fermentación $(8,9)$. No obstante, la hidrólisis con ácido diluido sigue siendo un método promisorio si se controlan las condiciones del tratamiento.

En este documento se presentan los resultados obtenidos al someter el raquis de banano (Cavendish Valery) a una hidrólisis con ácido sulfúrico (H2SO4) diluido, considerando la composición de holocelulosa, azúcares reductores y HMF según la severidad del tratamiento.

\section{MÉTODOS}

\section{ANÁLISIS COMPOSICIONAL}

Algunos análisis se hicieron de acuerdo a las normas estándar ASTM (American Society for Testing Materials). En la preparación de la biomasa para el análisis composicional se tomaron 10 raquis al azar, se fragmentó con un cuchillo, se secó en horno, aplicando la norma ASTM E 1757; la humedad siguiendo la norma ASTM E871, los extractivos con etanol con la ASTM
1690, el residuo insoluble en ácido y azúcares totales con la ASTM 1721 y la 1758 respectivamente, y usando la norma ASTM D1102, se determinó el porcentaje de cenizas.

Por su parte, el contenido de celulosa se determinó por el método de Seifert $(10,11)$; la determinación de holocelulosase realizó por el método del hipoclorito $(11,12)$; los azúcares reductores y disponibles se determinaron por el método de UV/Vis empleando el ácido 3,5-Dinitrosalić́lico (DNS) $(7,13)$, mientras que el HMF se determinó por el método de White $(14,15)$.

\section{HIDRÓLISIS CON ÁCIDO DILUÍDO}

\section{Hidrólisis en autoclave}

En una primer serie de ensayos se empleó ácido sulfúrico al $1 \%$, temperatura de 120 ㅇ y relación líquido-sólido de 8:1 (7). Para este ensayo se pesaron muestras de $10 \mathrm{~g}$ de vástago de banano (base seca y preparado se acuerdo a la norma ASTM E1757), se impregnaron con $80 \mathrm{~mL}$ de solución ácida, y luego se introdujeron en un autoclave a 120 oc (tiempo de elevación de 30 min hasta alcanzar la temperatura), 15 psi de presión. En periodos de $30 \mathrm{~min}, 60 \mathrm{~min}, 120 \mathrm{~min}$ y 180 min se tomaron muestras de hidrolizado y de la fracción sólida para determinar el contenido de azúcares reductores y HMF.

Para comparar el efecto de temperatura, $\mathrm{pH}$, y tiempo de los tratamientos sobre la composición de los AR, hemicelulosa, celulosa, materia seca y HMF; se empleó el factor de severidad (RO) y severidad combinada (CS) que se calcularon con las ecuaciones 1 y 2 respectivamente (9).

$$
\begin{gathered}
R_{0}=t \exp \left(\frac{T-100}{14,75}\right) \\
C S=\log R_{0}-p H
\end{gathered}
$$

En donde $t$ es el tiempo de residencia ( $\min ), T$ es la temperatura de reacción (으), y 100, la temperatura de referencia $(100 \stackrel{\circ}{ } \mathrm{C})$. El pH es determinado teóricamente (aplicando la fórmula $\mathrm{pH}=-\log [\mathrm{H}+]$ ) de acuerdo a la concentración de ácido sulfúrico añadido(9).

El empleo de la CS como parámetro de análisis en una hidrólisis ácida es considerado como el más adecuado y consistente hasta el momento (8) y por esta razón se empleó en esta investigación. 


\section{Hidrólisis en reactor de $50 \mathrm{~mL}$.}

Una segunda serie de experimentos se realizó a temperaturas más altas, para lo cual se usó un reactor de acero inoxidable de $50 \mathrm{~mL}$ de volumen interno, provisto de manómetro y válvula de seguridad, el cual era calentado externamente por una plancha de calentamiento de la marca Daigger y un baño de glicerina agitado magnéticamente a 200 rpm (Fig. 1a). La biomasa fue introducida al reactor en un recipiente de vidrio refractario como se muestra en la figura $1 \mathrm{~b}$ y con una relación sólido líquido 1:8, proporción que se obtuvo como se explica en la sección 3.2. La

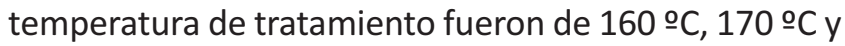
180 으, tomada en la pared externa del reactor. Estas temperaturas fueron calibradas antes de realizar la hidrólisis como se detalla en la sección 3.2.2.

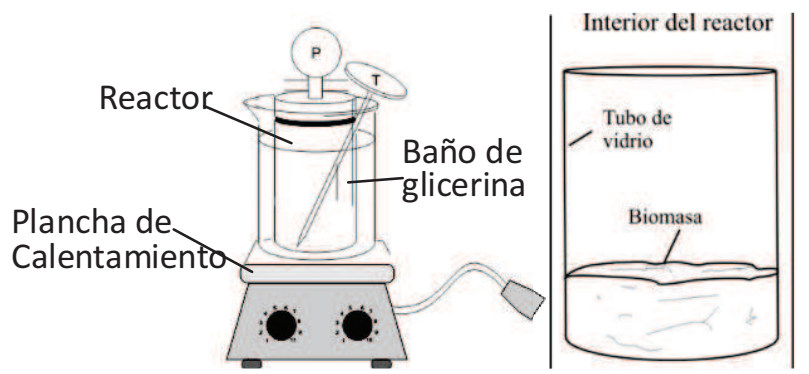

(a)

(b)

Figura 1. (a) Montaje para el calentamiento del reactor y (b) arreglo al interior del reactor.

Una vez la biomasa ha sido sujeto al tratamiento, se determinó el porcentaje de materia seca insoluble en agua (\% MS, ecuación 3), correspondiente al residuo remanente luego de que el material sólido tratado por hidrólisis ácida, es sometido a un lavado con agua destilada hasta que el pH del líquido retirado es neutro.

$$
\begin{aligned}
\% M S & =\frac{g M S}{\text { gbiomasa }} \\
& \times 100
\end{aligned}
$$

\section{RESULTADOS Y DISCUSIÓN}

\section{ANÁLISIS COMPOSICIONAL DEL RAQUIS DE BANANO}

Los resultados son basados en la composición en base seca y cada uno de los análisis se hizo por triplicado (Tabla 1).

Según los datos obtenidos, hay un contenido bajo de residuo insoluble en ácido, constituido principalmente por lignina (según la norma ASTM E 1721), si se compara con otro tipo de biomasa como la madera del pino que suele estar por encima del $20 \%$. Siendo la lignina un material de la pared celular, su presencia en concentraciones cada vez mayores, está relacionada inversamente con la accesibilidad de la holocelulosa al ataque químico o enzimático (2).

La holocelulosa, constituye una fuente potencialmente abundante de azúcares como se evidencia en la información suministrada en la tabla 1 . Adicional a la holocelulosa, la presencia de azúcares disponibles, aumenta la fuente de azúcares constituyendo en total, aproximadamente el $53 \%$ del raquis.

Tabla 1. Composición del vástago de plátano

\begin{tabular}{lc}
\hline \multicolumn{1}{c}{ Componente (método) } & Porcentaje (\%) \\
\hline Extractivos con etanol & 10,00 \\
Holocelulosa (hi poclorito) & 53,31 \\
Celulosa (Seifert) & 32,60 \\
Hemicelulosa (por difer encia) & 20,71 \\
Materia insoluble en ácido & 14.72 \\
Azúcares reductores & 0,052 \\
Azúcares disponibles & 1,86 \\
Cenizas & 12,00 \\
\hline
\end{tabular}

Referencia: los autores

Antes de tratar mediante la hidrólisis ácida a la biomasa, se quiso determinar la efectividad del método del DNS para la cuantificación de azúcares reductores. Asumiendo una eventual hidrólisis total de la biomasa, se cuantificó el contenido de azúcares reductores. Para esto, se procedió a realizar una hidrólisis total del material (basado en la norma ASTM 1758 y 1721) y la posterior determinación de los azúcares reductores, lo cual mostró resultados contradictorios, como se explica a continuación. El método del DNS se basa en la reacción de la glucosa con el reactivo de DNS y la medida de la absorbancia a $540 \mathrm{~nm}$. Éste monosacárido constituye la base de cálculo para el contenido de azúcares. Éste método de análisis fue usado para evaluar el contenido de azúcares reductores en varias investigaciones similares $(7,9)$ a la realizada en este estudio. Siendo éstas las circunstancias, se estableció que el contenido de carbohidratos totales de la biomasa del raquis fue de 344,5 +/- 5,0 mg, sin embargo, la cantidad de muestra tomada fue de solo $306,0 \mathrm{mg}$, habiendo una clara inconsistencia en el balance de masa. Para que éste análisis resulte en una sobre 
estimación pueden sugerirse varias razones: la formación de otros agentes reductores provenientes de la degradación de los diferentes constituyentes de la biomasa de identidad desconocida, a sustancias interferentes en la longitud de onda de cuantificación que no fueron tenidas en cuenta cuando el autor original desarrolló la técnica, o a que el contenido de hexosanos no es comparativa más grande que los otros carbohidratos comunes (pentosanos) como para establecer la glucosa componente predominante. Por lo tanto, el método del DNS no resulta adecuado para cuantificar el contenido de azúcares después de una hidrólisis ácida de la manera en la que aquí se ha hecho. Sin embargo, los resultados dados por el análisis aún pueden ser de utilidad y por eso, en este trabajo se usó para demostrar los avances en la reacción, entendiéndose esta medida como la concentración de agentes reductores del DNS (AR). El resto de los análisis, contenido de celulosa, hemicelulosa y 5-HMF sirvieron como evidencia adicional para evaluar el progreso de la degradación de la biomasa.

\section{HIDRÓLISIS CON ÁCIDO DILUIDO}

\section{Hidrólisis en autoclave}

Con el fin de conseguir una relación adecuada de líquido: sólido que garantice una solución de azúcares lo suficientemente concentrada al final de la hidrólisis que ha sido estimada por otros investigadores en un $10 \%$ (6), se tuvo en cuenta la cantidad máxima de azúcares reductores y cantidad mínima de líquido que impregna toda la biomasa, de la siguiente manera: Si se llega a una hidrólisis completa, los azúcares liberados, corresponderían a la suma de la cantidad de holocelulosa y de azúcares totales disponibles, es decir, aproximadamente el $53 \%$ de toda la biomasa. De otro lado, la cantidad de mínima de líquido está limitada por la capacidad de retención de agua de la biomasa de vástago de plátano. Para encontrar la cantidad mínima que impregna toda la biomasa, se pesó $1.00 \mathrm{~g}$ de biomasa seca y se le añadió un exceso de solución ácida, se mezcló y se dejo en reposo por $24 \mathrm{~h}$. Al final de este periodo, el material fue filtrado por gravedad hasta remover el exceso de líquido, luego se pesó nuevamente y se encontró que la cantidad de líquido retenido corresponde a aproximadamente $8 \mathrm{ml}$.

Una vez conocido el volumen de solución ácida que se adiciona a la biomasa se procedió a hacer la hidrólisis ácida. La tabla 2 muestra los resultados derivados del tratamiento del raquis. Los valores de factor de serveridad (Ro) y severidad combinada (CS) se calcularon con las ecuaciones 1 y 2 respectivamente.

Tabla 2. Resultados de los análisis después de hidrólisis ácida a $120^{\circ} \mathrm{C}$.

\begin{tabular}{lccccc}
\hline \multirow{2}{*}{ Análisis } & \multicolumn{5}{c}{ Tiempo (min) } \\
\cline { 2 - 6 } & $\mathbf{0}$ & $\mathbf{3 0}$ & $\mathbf{6 0}$ & $\mathbf{1 2 0}$ & $\mathbf{1 8 0}$ \\
\hline Ro & N/A & 135 & 233 & 27939 & 5028968 \\
CS & N/A & 1,08 & 1,38 & 1,67 & 1,85 \\
$A R(g / L)$ & 0,520 & 2,64 & 2,86 & 3,76 & 3,86 \\
HMF & \multicolumn{5}{c}{ No detectable } \\
\hline
\end{tabular}

Fuente: el autor.

El uso de la CS tiene la ventaja de integrar los efectos de la temperatura, $\mathrm{pH}$ y tiempo en un solo valor, que permite observación más clara de los resultados y su posterior análisis. En la gráfica de la figura 2 se aprecia la evolución en la concentración de AR primero rápidamente en la primera hora y luego cayendo la taza de producción, mientras que el figura 3 se muestra una correlación lineal cuando se emplea la CS contra AR. EI aumento en la concentración de AR puede deberse a la degradación de oligosacáridos o polisacáridos fácilmente hidrolizables que suelen hacer parte de la fracción de extractivos (tabla 1). También es posible que una ocurra degradación parcial en zonas de fácil acceso químico en las hemicelulosas. En cuanto a la celulosa, siendo un polímero más resistente al ataque químico, tiene una taza de degradación menor o quizás no ocurra en lo absoluto. Estos hechos son corroborados en los experimentos hechos a temperaturas más altas.

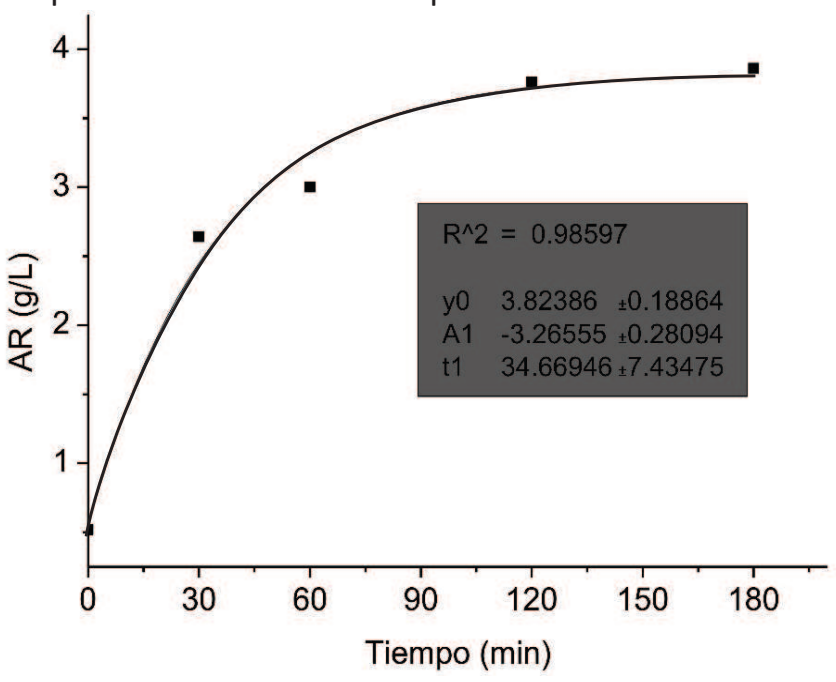

Figura 2.Concentración de agentes reductores (AR) en la hidrólisis ácida del vástago de banano. La línea de tendencia se ajustó con una ecuación exponencial con el programa origen 6. 


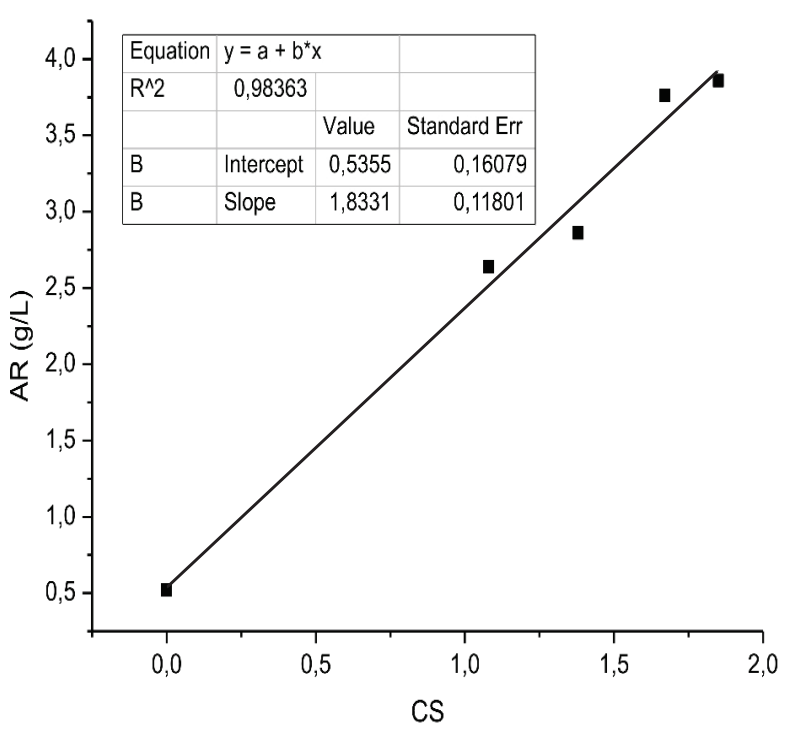

Figura 3. Contenido de azúcares reductores del hidrolizado en función de la severidad combinada. Ajuste lineal usando el programa origen 6.

El HMF puede estar presente si ocurre una degradación de monosacáridos. En el experimento a $120^{\circ} \mathrm{C}$, para todos los tiempos, la presencia de HMF no fue detectable ( $L D=0,0012 \mathrm{mg} / \mathrm{L}$, calculado como 10 desviaciones estándar, SD) mostrando que ésta sustancia no se forma bajo esas condiciones de severidad en el tratamiento.

Siendo la concentración de AR inferior al $10 \%$, una serie de nuevos experimentos fueron programados, en donde se tendrá en cuenta el efecto sobre el contenido de celulosa, hemicelulosa y la presencia de 5-HMF.

\section{Hidrólisis en reactor de $50 \mathrm{~mL}$}

Para establecer las condiciones de operación apropiadas de temperatura se programó un calentamiento a la máxima temperatura del agitador $\left(500{ }^{\circ} \mathrm{C}\right)$ y se fue regulando hasta alcanzar la temperatura de operación. Se encontró inicialmente que para obtener una temperatura de $160{ }^{\circ} \mathrm{C}$ en la pared del reactor, es necesario que la placa esté a 320 oc. Para las otras temperaturas se procedió de forma similar, modulando la temperatura según lo indicado por equipo. Para establecer un perfil de calentamento hasta alcanzar la temperatura de trabajo, entonces se registró la temperatura en la pared del reactor (TPR) cada dos minutos y así se obtuvo la gráfica de la figura 4.

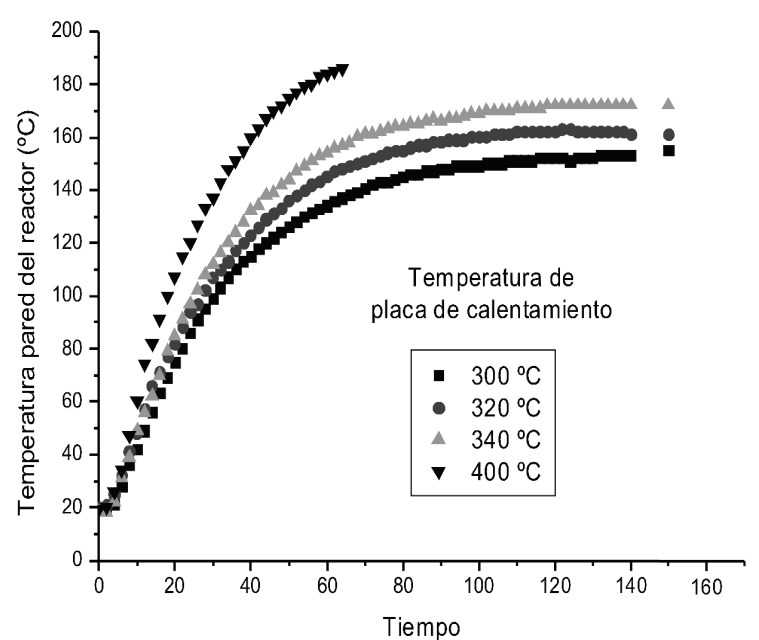

Figura 4. Acondicionamiento del reactor de hidrólisis ácida.

Puesto que la curva empieza a tener un comportamiento asintótico a los $150^{\circ} \mathrm{C}$ después de los $90 \mathrm{~min}$, se verificó la temperatura en un periodo adicional de $60 \mathrm{~min}$ con el fin de que se garantizara un tiempo de por lo menos 60 min (tiempo máximo en la hidrólisis ácida) de temperatura constante (intervalo de $+/ 3^{\circ} \mathrm{C}$ ). Luego de esto, se programó el equipo a $400^{\circ} \mathrm{C}$ para observar el comportamiento de temperatura. Puesto que después de alcanzar un tiempo de 64 min de calentamiento la presión empezó a rebasar el valor seguro (180 psi) se detuvo el calentamiento. De manera que, se hicieron dos nuevos experimentos con temperatura de la placa de $320^{\circ} \mathrm{C}$ y $340^{\circ} \mathrm{C}$ con lo cual se encontraron las temperaturas de operación de $160^{\circ} \mathrm{C}$ y 170 ㅇ $\mathrm{C}$ respectivamente después de los 90 min para todos los casos.

A pesar que las temperatura eran adecuadas, el tiempo de elevación fue extenso (90 min) comparado con el tiempo de tratamiento (máximo de $60 \mathrm{~min}$ ) por lo tanto se debió modificar la forma de operar el sistema. Para esto, se encontró que colocando la temperatura de placa a $340{ }^{\circ} \mathrm{C}, 360^{\circ} \mathrm{C}$ y $380^{\circ} \mathrm{C}$, y luego la introducción súbita del reactor en el baño, se llega a TPR de $160^{\circ} \mathrm{C}$, $170{ }^{\circ} \mathrm{C}$ y $180{ }^{\circ} \mathrm{C}$, en menos de $5 \mathrm{~min}$ según la temperatura de la placa. La gráfica de la figura 5 ilustra los hechos. 


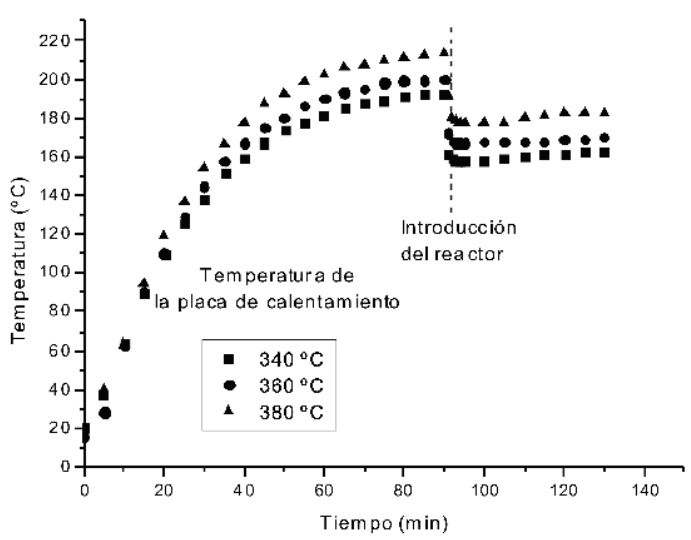

Figura 5. Acondicionamiento del reactor. Calentamiento e introducción del reactor en el baño de glicerina.
Una vez se calibró el funcionamiento del reactor de 50 $\mathrm{mL}$, el material lignocelulósico fue sometido a los diferentes tratamientos. Los efectos relacionados con los cambios químicos de la biomasa del raquis según la CS son mostrados en la tabla 3 y graficados como se muestra en la figura 6 .

De acuerdo al parámetro AR, se observa que el valor va aumentando hasta alcanzar un máximo de 32,4 g/L cuando la CS está entre 2,7 y 3,0. En el caso de las severidades combinadas de 0 a 2,0 se observa el mismo comportamiento lineal previamente observado para el tratamiento en autoclave (figura 3). Esta tendencia creciente en contenido de $A R$ se asocia a la sacarificación de sustancias fácilmente hidrolizables

Tabla 3. Datos de variación de la composición de la biomasa con respecto a la CS.

\begin{tabular}{ccccccccccc}
\hline No. & $\mathbf{C S}$ & $\begin{array}{c}\mathbf{T} \\
(\mathbf{o})\end{array}$ & $\begin{array}{c}\mathbf{t} \\
(\mathbf{m i n})\end{array}$ & $\begin{array}{c}\mathbf{C} \\
(\%)\end{array}$ & $\begin{array}{c}\text { \% } \\
\text { AR }\end{array}$ & $\begin{array}{c}\text { \% } \\
\text { MS }\end{array}$ & $\begin{array}{c}\% \\
\text { Cel }\end{array}$ & $\begin{array}{c}\text { HC } \\
\text { HM }\end{array}$ & $\begin{array}{c}\text { g/L } \\
\text { 5-HMF }\end{array}$ \\
\hline 1 & 0 & - & - & - & 0,52 & 100 & 31,6 & 53,3 & 21,7 & ND \\
2 & 0,474 & 160 & 10 & 0,05 & 2,03 & 67,5 & 46,7 & 67,6 & 20,9 & ND \\
3 & 0,775 & 160 & 20 & 0,05 & 3,7 & 62,4 & 48,2 & 69,9 & 21,7 & ND \\
4 & 1,07 & 160 & 40 & 0,05 & 3,91 & 60,0 & 53,4 & 75,9 & 22,5 & ND \\
5 & 1,07 & 170 & 20 & 0,05 & 3,47 & 60,0 & 51,3 & 73,6 & 22,3 & ND \\
6 & 1,67 & 180 & 40 & 0,05 & 5,14 & 63,6 & 56,7 & 74,3 & 17,6 & ND \\
7 & 1,78 & 160 & 10 & 1,0 & 5,84 & 65,6 & 48,9 & 72,6 & 23,7 & ND \\
8 & 2,07 & 170 & 10 & 1,0 & 10,2 & 62,0 & 50,0 & 73,1 & 23,1 & ND \\
9 & 2,08 & 160 & 20 & 1,0 & 14,0 & 55,8 & 53,7 & 72,3 & 18,6 & ND \\
10 & 2,38 & 160 & 40 & 1,0 & 23,9 & 47,5 & 64,7 & 73,5 & 8,8 & ND \\
11 & 2,67 & 170 & 40 & 1,0 & 25,7 & 46,3 & 69,0 & 72,4 & 3,4 & ND \\
12 & 2,77 & 170 & 50 & 1,0 & 31,0 & 47,4 & 65,3 & 72,0 & 6,7 & $7,06 \times 10^{-4}$ \\
13 & 2,97 & 180 & 40 & 1,0 & 32,4 & 48,4 & 66,6 & 72,6 & 6,0 & $9,06 \times 10^{-4}$ \\
14 & 3,06 & 180 & 50 & 1,0 & 31,6 & 44,7 & 67,5 & 68,8 & 1,3 & 0,0307 \\
15 & 3,36 & 180 & 50 & 2,0 & 21,6 & 32,2 & 73,4 & 74,0 & 0,6 & 0,503 \\
16 & 3,62 & 180 & 60 & 3,0 & 15,3 & 24,3 & 80,5 & 81,1 & 0,6 & 3,44 \\
\hline
\end{tabular}

CS: Severidad Combinada; T: temperatura; C: Concentración de ácido sulfúrico, AR: Azúcares Reductores; MS: Materia Seca; Cel: Celulosa; HC: Holocelulosa; HM: Hemicelulosa. 5-HMF: 5-hidroximetilfurfural, ND: no detectable. * AR de la fracción líquida remanente después de hidrólisis.

como el almidón por ejemplo, y la hemicelulosa como se aprecia en la gráfica cuyo contenido evoluciona de manera inversa al contenido de AR. Después de rebasar la $\mathrm{CS}=3,0$ ocurre un rápido decaimiento en el contenido de AR al mismo tiempo que el HMF se va generando. Estos hechos al ser contrastados, sugieren la degradación de los monosacáridos recién formados.

El contenido de celulosa al parecer permanece constante entre las $\mathrm{CS}=1,0$ y $\mathrm{CS}=3,0$; mientras que la concentración de holocelulosa va en aumento hasta ser similar a la de la celulosa, lo que resulta plausible si se considera que la hemicelulosa es el material más fácilmente degradable bajo estas condiciones. De hecho, al observar el contenido de celulosa en la materia seca (Contenido de celulosa $=$ MS $\times \%$ Celulosa/100) con respecto al valor medio (Contenido inicial de celulosa $=31,6 \%$ ) son pocos los datos que alejan más de una desviación estándar (SD) con respecto a la media (Figura 7) y exceptuando los datos 6 , 
15 y 16 (Tabla 2), el resto están dentro de +/ 2SD. Por lo tanto, se puede inferir que no hay una variación significativa en la cantidad de celulosa para valores de severidad de 0 a 3,06, mientras que por encima de este valor el contenido cambia rápidamente indicando que la celulosa se está degradando. A pesar de que se verifica la degradación de celulosa, los productos formados probablemente se degradan, pues no se observó un aumento en el contenido de AR sino por el contrario, una rápida disminución coincidente con la formación del producto de degradación de hexosas HMF como se mencionó anteriormente.

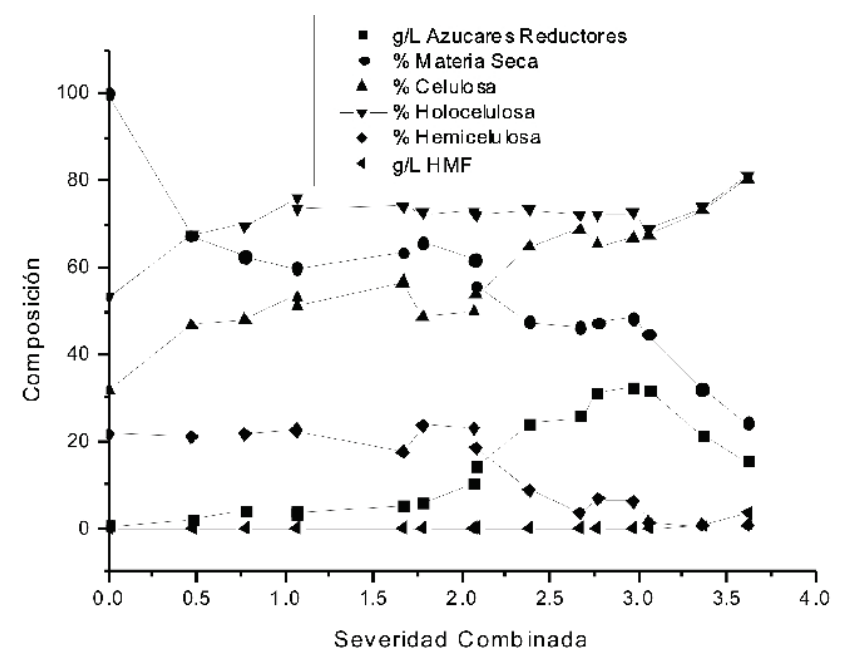

Figura 6. Variación en la composición de la biomasa después del tratamiento con $\mathrm{H}_{2} \mathrm{SO}_{4}$ diluído.

El HMF ha sido designado como la toxina clave en los hidrolizados lignocelulósicos pues afecta la taza de fermentación cuando se emplea levaduras (16). En relación a esto, Keating, Panganiban et al. (17) encontraron que en la etapa de fermentación, el HMF muestra un efecto desfavorable sobre el consumo de hexosas por parte de la levadura Sccaharomyces cerevisiae en la etapa de fermentación cuando las concentraciones son de $0,6 \mathrm{~g} / \mathrm{L}$ y $0,8 \mathrm{~g} / \mathrm{L}$ y esto se refleja en un producción desacelerada de etanol. Siendo esta la perspectiva, el tratamiento de CS $=3,62$ resulta inadecuado, no solo por la producción disminuida de azúcares sino por la acelerada formación de HMF.

De otro lado, al hacer un cálculo del contenido de holocelulosa (Contenido de holocelulosa $=$ MS $\times$ \%Holocelulosa / 100) es posible establecer que el contenido de holocelulosa si varía sustancialmente disminuyendo, hasta ser similar al contenido de celulosa (Figura 8). Con esto se constata, que la hemicelulosa es el material que se degrada progresivamente hasta casi desaparecer cuando la severidad combinada es de alrededor de 3,0. Este hecho se observa claramente cuando se grafica la CS contra el contenido de hemicelulosa en la figura 8, además otros autores han reportado el mismo comportamiento en severidades que oscilan entre $1,9 \mathrm{y}$ 2,7 (8) por lo que se puede asegurar que el tratamiento, debido a la colapso estructural provocado por la pérdida de hemicelulosa, eventualmente puede facilitar la degradación de la celulosa por vía enzimática.

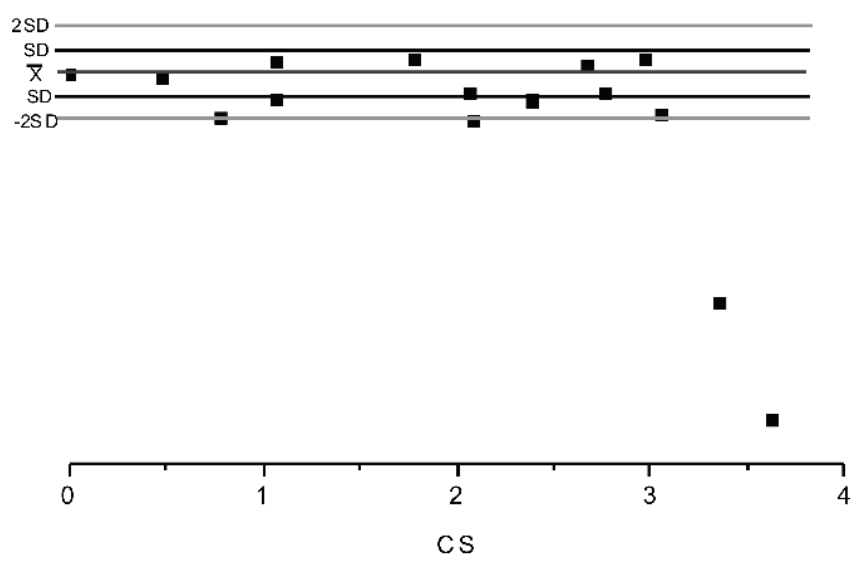

Figura 7. Valoración estadística del contenido de celulosa con respecto a la CS. SD=0,8083 y para la media de $31,6 \%$ de contenido de celulosa.

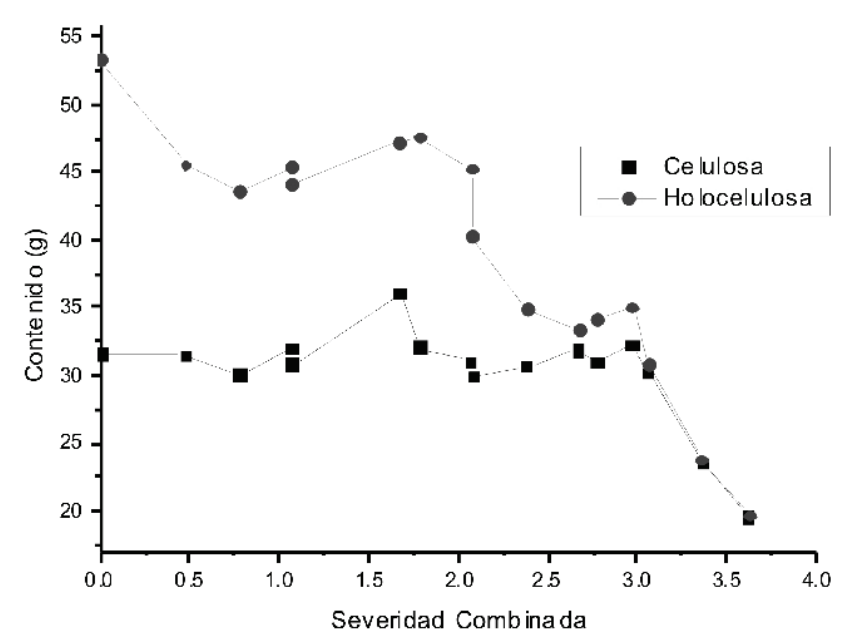

Figura 8. Variación en la cantidad de celulosa y holocelulosa por cada $\mathbf{1 0 0} \mathrm{g}$ de hidrolizado

Rev. Invest. Univ. Quindío (22): 83- 91. Armenia - Colombia 


\section{CONCLUSIONES Y RECOMENDACIONES}

La Severidad Combinada (CS) como factor comparativo entre diferentes tratamientos térmicos ha resultado ser una herramienta útil en esta investigación y hasta el momento no existe un parámetro mejor que permita un acercamiento más sencillo al estudio de las degradaciones que ocurren en la biomasa(8). Los resultados aquí mostrados, aunque muestran variaciones no correlacionadas de forma lineal entre la CS y la concentración de AR, hemicelulosa y celulosa, si muestra tendencias lo suficientemente explícitas como para tomar decisiones frente a lo que está sucediendo con los principales componentes de la biomasa.

En esta investigación se logró establecer por primera vez, que sucede con la hemicelulosa y celulosa luego de que la biomasa del raquis de banano fuera sometida a una hidrólisis con ácido sulfúrico diluído. La hemicelulosa es degrada a partir de Severidad Combinada (CS) de 2,0 hasta su totalidad luego de que las condiciones de tratamiento supera una CS de 3,0. Por su parte, la celulosa, muestra evidencia de degradación solo después de que la CS es superior a 3,0.
Aunque en este estudio de hidrólisis ácida se constata la degradación de hemicelulosa sin producción de HMF a CS de 2,67 , la concentración de AR $(25,7 \mathrm{~g} / \mathrm{L})$ no es lo suficientemente alta como para justificar una fermentación económicamente viable. Sin embargo, el contenido remanente de celulosa, debido a que se encuentra libre de hemicelulosa, posibilitaría su digestión por métodos enzimáticos, por lo que se constituye en una fuente adicional de azúcares fermentables lo que se constituye en el siguiente paso a investigar.

\section{RECONOCIMIENTO}

Este artículo hace parte de los resultados del proyecto de investigación No. 463 "Obtención de alcohol carburante a partir del pseudotallo de plátano y banano" financiado por la Universidad del Quindío a través de la Vicerrectoría de Investigaciones, y los autores son investigadores en el laboratorio de química industrial y del grupo de investigación QIDEA (Grupo Químico de Investigación y Desarrollo Ambiental, categoría Cen COLCIENCIAS).

\section{BIBLIOGRAFÍA}

1. Xu J, Thomsen MH, Thomsen AB. Enzymatic hydrolysis and fermentability of corn stover pretreated by lactic acid and/or acetic acid. Journal of Biotechnology. 2009;139:300-5.

2. Hendrics ATWM, Zeeman G. Pretreatments to enhance the digestibility of lignocellulosic biomass. Bioresource Technology. 2009;100:10-8.

3. Zuluaga R, Putaux J-L, Restrepo A, Mondragon I, Gañán P. Cellulose microfibrils from banana farming residues: isolation and characterization. Cellulose. 2007;14:585-92.

4. Guarnizo-Franco A, Martínez-Yepes PN, Valencia HA. Pretratamientos de la celulosa y biomasa para la sacarificación. Scientia et Technica. 2008;42:284-9.

5. Guarnizo-Franco A, Martínez-Yepes PN. Experimentos de química orgánica con enfoque en ciencias de la vida. Armenia: Elizcom; 2009.

6. Yang B, Wyman CE. Pretreatment: the key to unlocking low-cost cellulosic ethanol. Biofuels, Bioproducts and Biorefining. 2008;2:26-40.

7. Zhao X, Zhang L, Liu D. Comparative study on chemical pretreatment methods for improving enzymatic digestibility of crofton weed stem. Bioresource Technology. 2008; 993729-36.

8. Pedersen $\mathrm{M}$, Meyer AS. Lignocellulose pretreatment severity - relating $\mathrm{pH}$ to biomatrix opening. New Biotechnology. 2010;27(6):739-50. 
9. Larsson S, Palmqvist E, Hahn-Ha"gerdal Br, Tengborg C, Stenberg K, Zacchi G, et al. The generation of fermentation inhibitors during dilute acid hydrolysis of softwood. Enzyme and Microbial Technology. 1999;24:151-9.

10. Seifert K. Zur fellulose-Schnellbestimmung nach der acetylation-methode. Das papier. 1960;14(3):104.

11. Butt DAE. Formation of phenols from the low-temperture fast pyrolysis of Radiata pine (Pinus radiata) Part I. Influence of molecular oxygen. J Anal Appl Pyrolysis. 2006;26(2006):38-47.

12. Norman AG, Jenkins SH. A new method for determination of cellulose, based upon observations on the removal of lignin and other encrusting materials. Biochemical Journal. 1933;27:818-31.

13. James CS. Analytical Chemistry Of Foods Book CHFS, editor. New York: Springer; 1998.

14. Zappalà M, Fallico B, Arena E, Verzera A. Methods for determination of HMF in honey: a comparison. Food control. 2005;16(2005):273-7.

15. Chi C, Zhang Z, Chang H-m, Jameel H. Determination of furfural and hydroxymethylfurfural formed from biomass under acidic conditions. Wood Chemistry and Technology. 2009;29:265-76.

16. Heer $D$, Sauer U. Identification of furfural as a key toxin in lignocellulosic hydrolysates and evolution of a tolerant yeast strain. Microbial Biotechnology. 2008;1(6):497-506.

17. Keating JD, Panganiban C, Mansfield SD. Tolerance and adaptation of ethanologenic yeasts to lignocellulosic inhibitory compounds. Biotechnology and Bioengineering. 2006;93(6):1196-206. 